\title{
Continuous decoding of grasping tasks for a prospective implantable cortical neuroprosthesis
}

\author{
Jacopo Carpaneto ${ }^{1 *}$, Vassilis Raos ${ }^{2,3}$, Maria A Umiltà ${ }^{4,5}$, Leonardo Fogassi ${ }^{4,5,6}$, Akira Murata ${ }^{7}$, Vittorio Gallese ${ }^{4,5}$ \\ and Silvestro Micera ${ }^{1,8}$
}

\begin{abstract}
Background: In the recent past several invasive cortical neuroprostheses have been developed. Signals recorded from the motor cortex (area MI) have been decoded and used to control computer cursors and robotic devices. Nevertheless, few attempts have been carried out to predict different grips.

A Support Vector Machines (SVMs) classifier has been trained for a continuous decoding of four/six grip types using signals recorded in two monkeys from motor neurons of the ventral premotor cortex (area F5) during a reach-to-grasp task.
\end{abstract}

Findings: The results showed that four/six grip types could be extracted with classification accuracy higher than $96 \%$ using window width of $75-150 \mathrm{~ms}$.

Conclusions: These results open new and promising possibilities for the development of invasive cortical neural prostheses for the control of reaching and grasping.

Keywords: Ventral premotor cortex (area F5), Brain decoding, Grasping, Neuroprosthesis

\section{Findings}

\section{Introduction}

In the recent past many efforts have been devoted to develop artificial devices to restore sensorimotor functions in people who lost them due to amputation, spinal cord injury, stroke, etc. [1-3]. The possibility of connecting the peripheral and central nervous system with artificial devices by means of invasive neural interfaces [1-5] is currently investigated in order to increase the number of possible functional connections between patients with impaired sensory/motor functions and the artificial device (e.g., hand prostheses, robotic arm, etc.) and to control it in a simple and intuitive way. Electrodes have been implanted invasively a) in peripheral nerves to achieve a bi-directional control of hand prostheses in amputees $[6,7] ; b)$ in the cortex, to extract user's motor commands from movement-related cortical signals $[2,8,9]$ or to deliver a sensory feedback by stimulating selected sectors of the somatosensory cortex [10]. Thus, invasive cortical neural prostheses (ICNPs) could help

\footnotetext{
* Correspondence: j.carpaneto@sssup.it

${ }^{1}$ Neural Engineering Area, The BioRobotics Institute, Scuola Superiore

Sant'Anna, Pisa, Italy

Full list of author information is available at the end of the article
}

subjects affected by several deficits caused by spinal cord injury, stroke, amyotrophic lateral sclerosis, cerebral palsy, and multiple sclerosis to re-establish some degree of autonomy by controlling an output on a computer or a robotic system. In most cases, research groups have focused their efforts on the extraction of information from the motor cortex (area M1) to drive a robotic arm: signals recorded from ensembles of M1 cortical neurons have been processed through different algorithms to predict reaching directions or trajectories of a robotic arm end-effector [11-14]. This approach has been tested with very promising results in animal models $[2,12]$ and recently in selected highly disabled subjects $[8,9,15]$. Moreover, individual [16] or ensemble M1 neurons data [17] have been used in order to predict hand or forearm muscle activity in brain controlled functional electrical stimulation (FES).

The situation becomes more challenging when several degrees of freedom need to be controlled (e.g., dexterous hand prostheses). In this case, even if recent results have shown that information related to finger movements can be obtained from M1 activities [18,19], it seems quite difficult to extract simultaneously the kinematics of all the fingers using this kind of approach. Therefore, the
C Biomed Central

(c) 2012 Carpaneto et al.; licensee BioMed Central Ltd. This is an Open Access article distributed under the terms of the Creative Commons Attribution License (http://creativecommons.org/licenses/by/2.0), which permits unrestricted use, distribution, and reproduction in any medium, provided the original work is properly cited. 
possibility of decoding higher level information (e.g., the specific grip type instead of the trajectories of the hand joints) may offer several advantages. Previous experiments have shown that neurons of ventral premotor area F5 (located in the posterior bank of the inferior limb of the arcuate sulcus and the cortical convexity immediately adjacent to it) do not specify/encode a given pattern of movements (as M1 neurons), but rather an end-state, like the goal of a motor act (e.g., grasping as a whole) [20]. Motor neural activity, recorded using single microelectrode [21], multi electrode array [22], ECoG [23], and Local Field Potentials [24], changes according to the properties of the object to be handled and, thus, of the grasping task. Recent studies demonstrated that it is possible to distinguish between power vs. precision grip $[22,23]$ or up to six grips considering different duration periods of the whole movement phase [25]. Starting from this neurophysiological rationale, a pattern recognition algorithm for a continuous decoding of four/six grip types from F5 neurons during execution of a reachto-grasp task is presented in this manuscript.

\section{Materials and methods}

Data analyzed in this paper have been collected from area F5 in the posterior bank of the inferior limb of the arcuate sulcus in three hemispheres (contralateral to the moving forelimb) of two awake monkeys (Macaca nemestrina). Behavioral apparatus and task, animals training and data collection have been described in [21]. Briefly, the monkey seated in front of a rotating turntable subdivided into six sectors, each containing a different object. The monkey had to fixate one object and press a key then release it, reach for and grasp the object, pull it, hold it and release it. The different objects were presented to the monkey in random order (8 repetitions for each object). Two sets of six geometric objects eliciting different grip types were used (i.e., original and special set composed by objects differing in size and shape, see Table 1 and [21]).

The dataset used in this paper consisted of 46 F5 neurons that were classified as purely motor grasping neurons. The activity of these neurons was not related to individual finger movements, but to the grasping action as a whole [21]. Thirty-six were tested with the objects of the original turntable and 10 with the objects of the special turntable. Properties of the neurons have been analyzed in [21] whereas the results of grips classification during different duration periods of the movement phase have been reported in [25].

\section{Decoding algorithm}

In order to investigate whether a reliable grip classification can be obtained during the object presentation and movement phase, normalized firing rate (nFR) have been extracted from F5 motor units during a period that goes from $1800 \mathrm{~ms}$ before to $200 \mathrm{~ms}$ after the key release event (e.g., starting of the movement phase). In particular, nFR was extracted for each unit of the F5 population and in each trial using analysis windows varying in duration (bin widths from 25 to $200 \mathrm{~ms}$ ) which progressively slid over the reference period with a moving step of $10 \mathrm{~ms}$. Normalization was done with respect to the maximum nFR among all trials. The F5 neural population response onset $\left(t_{\text {onset }}\right)$ has been detected using a threshold algorithm [26]. The onset corresponded to the moment when the F5 population nFR in an analysis window was more than 2.5 of standard deviation value, calculated over the first $400 \mathrm{~ms}$ of the object presentation phase (from -1800 to $-1400 \mathrm{~ms}$ ). The sub-threshold activity before key release (object presentation) was considered as baseline. The baseline and the above-threshold activity recorded during execution of the different grips were labeled and used as examples to train the classifier or to test its generalization skills. nFR of the different neurons have been classified using SVMs ( $v$-SVMs with radial basis kernel function) making use of the open source library LIBSVM [27]. Training and cross-validation has been done splitting data by trials and using a random selection of $25 \%$ of the trials for the training of the classifier and the remaining $75 \%$ of the trials for the testing. Two different classification methods have been used: i) direct discrimination of 5/7 classes (baseline plus four/six grip types); and ii) hierarchical discrimination (baseline versus above-threshold activity and then selection of four/six grip types). In the case of four objects classification, the first three object of the original set (i.e., cube, sphere, and cone) as well as the first three object of the special set (sphere in groove, large cylinder

Table 1 The objects of the original and special set and the grips used by the monkeys during grasping [21,25]

\begin{tabular}{lcccc}
\hline Original set & Grip type & $\#$ & Special set & Grip type \\
\hline Cube & Side grip & 1 & Sphere in groove & Advanced precision grip \\
Sphere & Side grip & 2 & Large cylinder in container & Finger prehension with thumb opposition \\
Cone & Side grip & 3 & Small sphere & Large sphere grip \\
Plate & Primitive precision grip & 4 & Small ring \\
Cylinder & Finger prehension & 5 & Large ring & Hook grip (index) Hand prehension \\
Ring & Hook grip (index) & 6 & Hook grip (4 fingers)
\end{tabular}


in container, and small sphere) have been clustered together because the grips used for their prehension shared common features (i.e., side grip or thumb/finger opposition) $[21,25]$. The accuracy of the objects classification has been assessed using a recognition ratio (RR), defined as the proportion of the grips correctly identified with respect to those classified.

\section{Statistical analysis}

A Friedman test $(p \leq 0.01)$ has been used to compare the results obtained from (i) the neurons tested with the two sets of objects (original vs special) and (ii) the two classification schemes (direct vs hierarchical discrimination). Moreover, a Kruskall-Wallis test $(\mathrm{p} \leq 0.01)$ has been used to verify the influence of the different bin-widths in the classification accuracy.
Results

In Figure 1, the mean nFR of the F5 motor neurons population for each object of the normal and special set is given. The response has been plotted from $1800 \mathrm{~ms}$ before to $1000 \mathrm{~ms}$ after the onset of the movement (the key release event that corresponds to $0 \mathrm{~ms}$ ). The key release event corresponds to $0 \mathrm{~ms}$ ). The mean $t_{\text {onset }}$ and the mean end of the movement $\left(t_{\text {mov }}\right)$ are marked as red and green horizontal line, respectively.

In the case of the original set of objects, the onsets of the neural population response, calculated with a threshold method (see Materials and methods section), preceded the start of movement whereas, in the case of special set of objects, the onsets were either preceding or following the start of the movement. $t_{\text {mov }}$ was $334 \pm 113 \mathrm{~ms}$ for the original set of objects and $407 \pm 192 \mathrm{~ms}$ for the special set of objects. In both cases, the prediction of grip type was
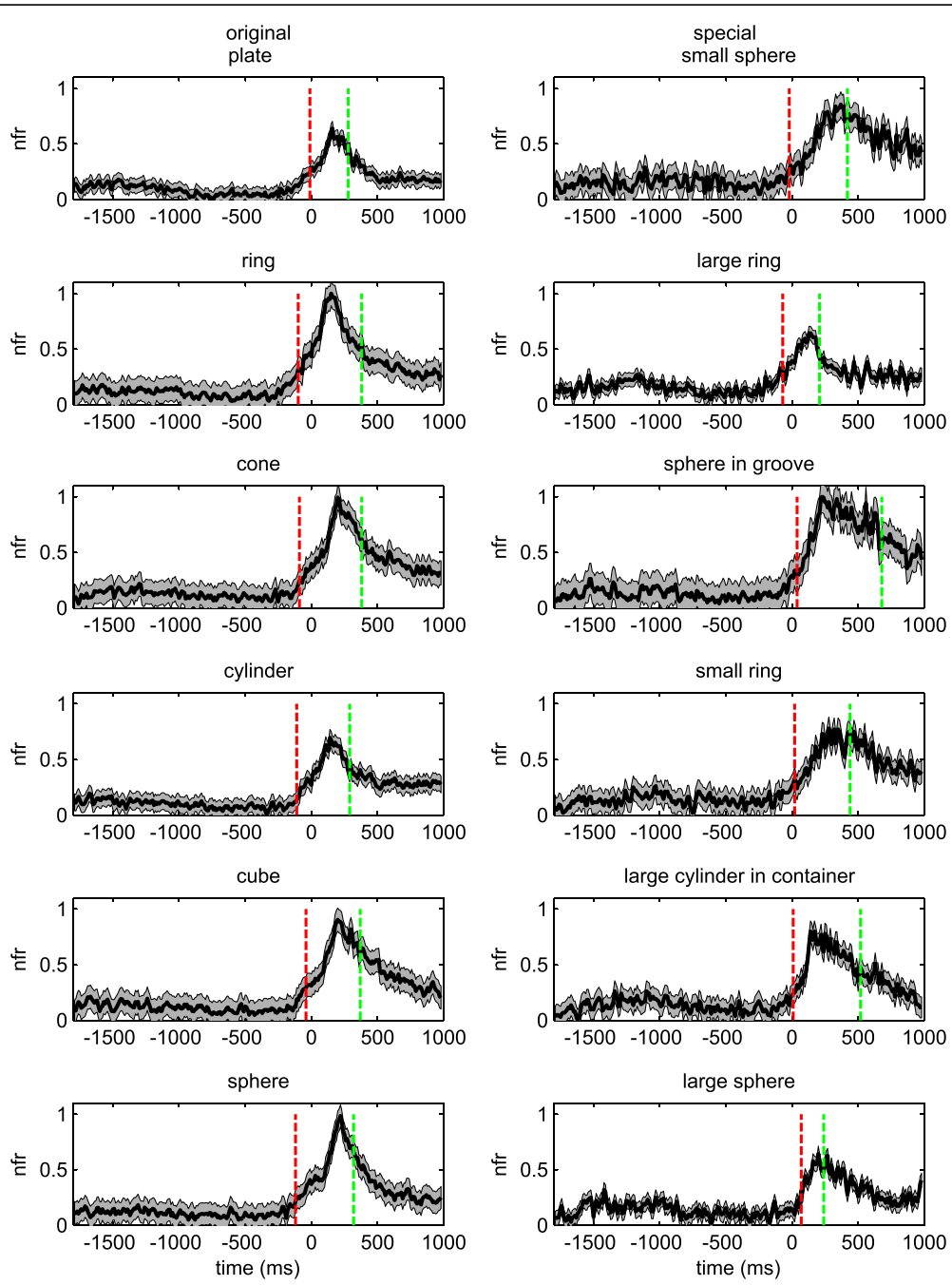

Figure 1 The mean normalized firing rate $(\mathrm{nFR}) \pm$ standard deviation, during grasping of different objects, of the F5 neurons tested with the original (left) and the special (right) set of objects (bin width $=\mathbf{2 5} \mathbf{~ m s ) . ~ t ~ = 0 ~ c o r r e s p o n d s ~ t o ~ t h e ~ k e y ~ r e l e a s e ~ e v e n t ~ ( s t a r t ~ o f ~ t h e ~}$ movement phase). The mean $t_{\text {onset }}$ and $t_{\text {mov }}$ for each object are indicated by red and green vertical lines. 
done in the first $200 \mathrm{~ms}$ of the movement phase well before its end.

The results of the classification as a function of the window width (from 25 to $200 \mathrm{~ms}$ ) are given in Figure 2 for all the different classification analyses (four or six grips, direct or hierarchical, original or special set).

Window widths between 75-150 ms seem to be sufficient to obtain the highest values of stable RR. More specifically, with the original set of objects and a window widths of $100 \mathrm{~ms}$, RR values were $99.11 \pm 0.52$ (direct) and $98.70 \pm 1.16$ (hierarchical) for the classification of 4 grips and $96.62 \pm 1.16$ (direct) and $95.74 \pm 1.32$ (hierarchical) for the classification of 6 grips. With the special set of objects and a window width of $150 \mathrm{~ms}$, RR values were $97.79 \pm 1.01$ (direct) and $97.30 \pm 1.17$ (hierarchical) for the classification of 4 grips and $97.18 \pm 0.95$ (direct) and $96.51 \pm 1.09$ (hierarchical) for the classification of 6 grips.

The performance of the two populations of neurons was significantly different $(\mathrm{p}<0.01)$ and this is likely due to the number of neurons belonging to each population (i.e., 36 and 10 neurons tested with the original and the special set of objects, respectively). Nevertheless, the differences between the mean accuracy obtained from the neurons tested with the original and special set of objects were limited (direct classification of 4 and 6 grips: $3.14 \%$ and $2.31 \%$, respectively; hierarchical classification of 4 and 6 grips: $4.03 \%$ and $2.88 \%$, respectively). These differences decreased below $2 \%$ using bins greater than $150 \mathrm{~ms}$. Concerning the classification schemes, even if the direct approach was significantly better than the hierarchical one $(\mathrm{p}<0.01)$, the differences between the two schemes were less than $2 \%$ (1\% using window width greater than $125 \mathrm{~ms}$ ). Finally, window length influenced significantly the accuracy $(\mathrm{p}<0.01)$. The use of

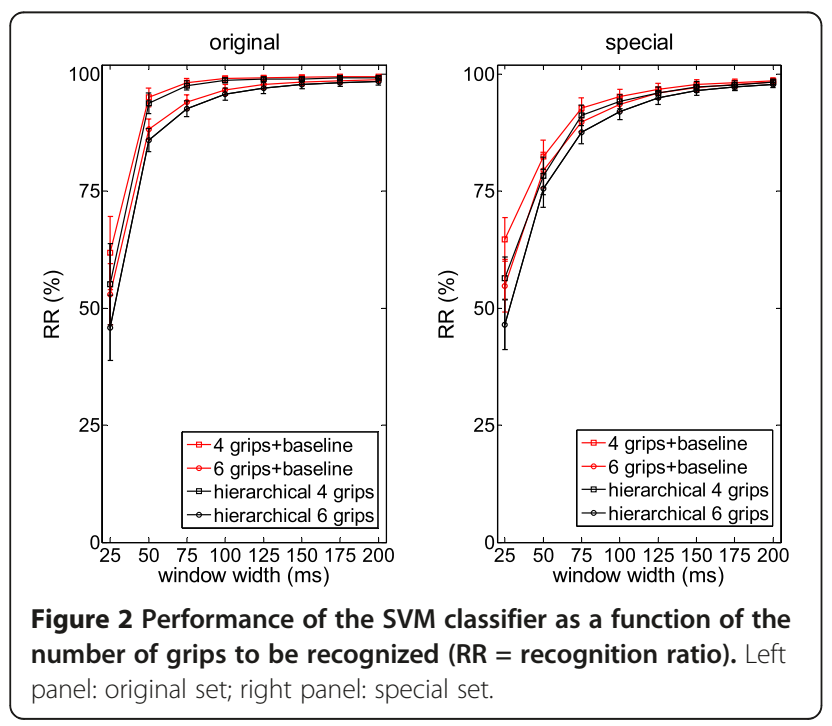

small bins $(25 \mathrm{~ms})$ resulted in deteriorated performance (i.e., differences worse than $30 \%$ as compared to the mean accuracy obtained with the use of $100 \mathrm{~ms}$ bins) whereas the use of bins larger than $150 \mathrm{~ms}$ did not result in any significant improvement (i.e., $0.34 \%$ and $0.72 \%$ differences in the mean accuracy between bins of 150 and $200 \mathrm{~ms}$ with the original and the special set of objects, respectively).

An example of the classification accuracy during the direct discrimination of $5 / 7$ classes obtained with a $100 \mathrm{~ms}$ window width is given in Figure 3.

Actual grips are represented by black ovals whereas predicted grips are represented by red crosses. Correct classification results in a superimposition between actual and predicted grips (i.e., black ovals and red stripes). Isolated red crosses represent errors made by the classifier and are plotted at the level of the predicted class. Most of the errors occur during the classification of 6 grips. In the case of the original set, the classifier assigns part of the features extracted during the grasping of objects 1 and 3 to grip 2, confirming the fact that objects 1,2 , and 3 are grasped in a similar way [21]. For the special set of objects, a similar behavior occurs for objects 1 and 2 and for the objects 5 and 6 (hook grip) [21].

\section{Discussion}

The extraction of grip types (e.g., precision grip, finger prehension, whole hand prehension) from F5 could be a very attractive solution to be applied complementary to the M1-based ICNPs. In fact, if the goal of the ICNP is

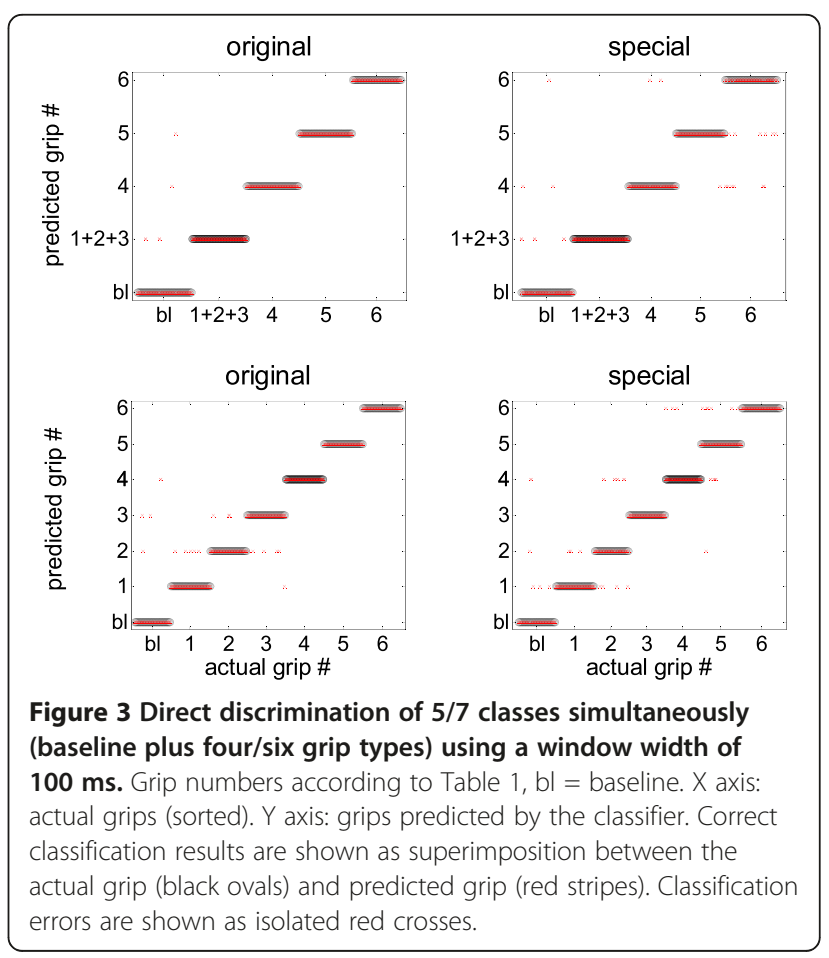


to control both reaching and manipulation of a dexterous arm-hand artificial robot, signals recorded from M1 can be used to decode information about the reaching phase and grasp timing (as already proved by the very interesting results achieved so far $[13,14]$ ) while F5 signals can be used for the detection of the desired grasping task (see Figure 4).

Recent papers [22,23] demonstrated the possibility to distinguish between precision and power grips in a reliable way whereas in [25] the decoding of 4-6 grips during the reaching phase has been analyzed in different normalized windows (e.g., from $25 \%$ to $100 \%$ of the reaching phase).

In this paper, an SVM classifier has been used to continuously predict different grips ( 4 and 6 ) from the activity of F5 motor neurons recorded during the reach to grasp task. This classifier was already used in a previous paper from our group for the decoding of different grips [25]. However, in the current paper a popula tion of purely motor neurons was analyzed whereas in [25] a population of motor and visuomotor units was employed. Secondly, in the present paper we investigated the effect of using different time windows while in the previous work one fixed window was used. As shown in Figure 1, activity of F5 neurons precedes or is contemporaneous to the onset of the movement. During hand transport phase and using a continuous classification scheme, it is possible to predict four/six grips with high $\mathrm{RR}$ using firing rate activity calculated over bins of $75 \mathrm{~ms}$ (for the original set of objects) and $150 \mathrm{~ms}$ (for the special set of objects). These results seem to be compatible with a real-time control of manipulation tasks (i.e., reaching and grasping) performed with ICNPs. In fact, the delay introduced by the grip decoding is similar to the maximum values proposed in case of an EMG-based control of prostheses (e.g., 100-125 ms [29]). motor related discharge, may allow the correct discrimination among grips even earlier than that reported in the present study $[21,25]$.

It must be taken into account that the decoding algorithm has been tested offline with single cell recordings. Real-time ICNPs with chronic multi-electrode arrays may perform worse, even if this limitation could be reduced thanks to the progress of micro and nanotechnologies [4] and the learning-induced tuning of the cells during real-time experiments [30]. Another issue is the limited number of decoded grips. Nevertheless, four or six grips should ensure a good grasping dexterity and a significant increase in the number of possible activities of daily living to be carried out.

In conclusion, a SVM based algorithm has been used for a continuous decoding of grip types from F5 motor neurons during execution of reach-to-grasp tasks. The results obtained show that four/six grip types were extracted with classification accuracy higher than 96\% using window width of 75-150 ms. These results introduce new and promising scenarios for the development of ICNPs. In fact, the possibility not only to control

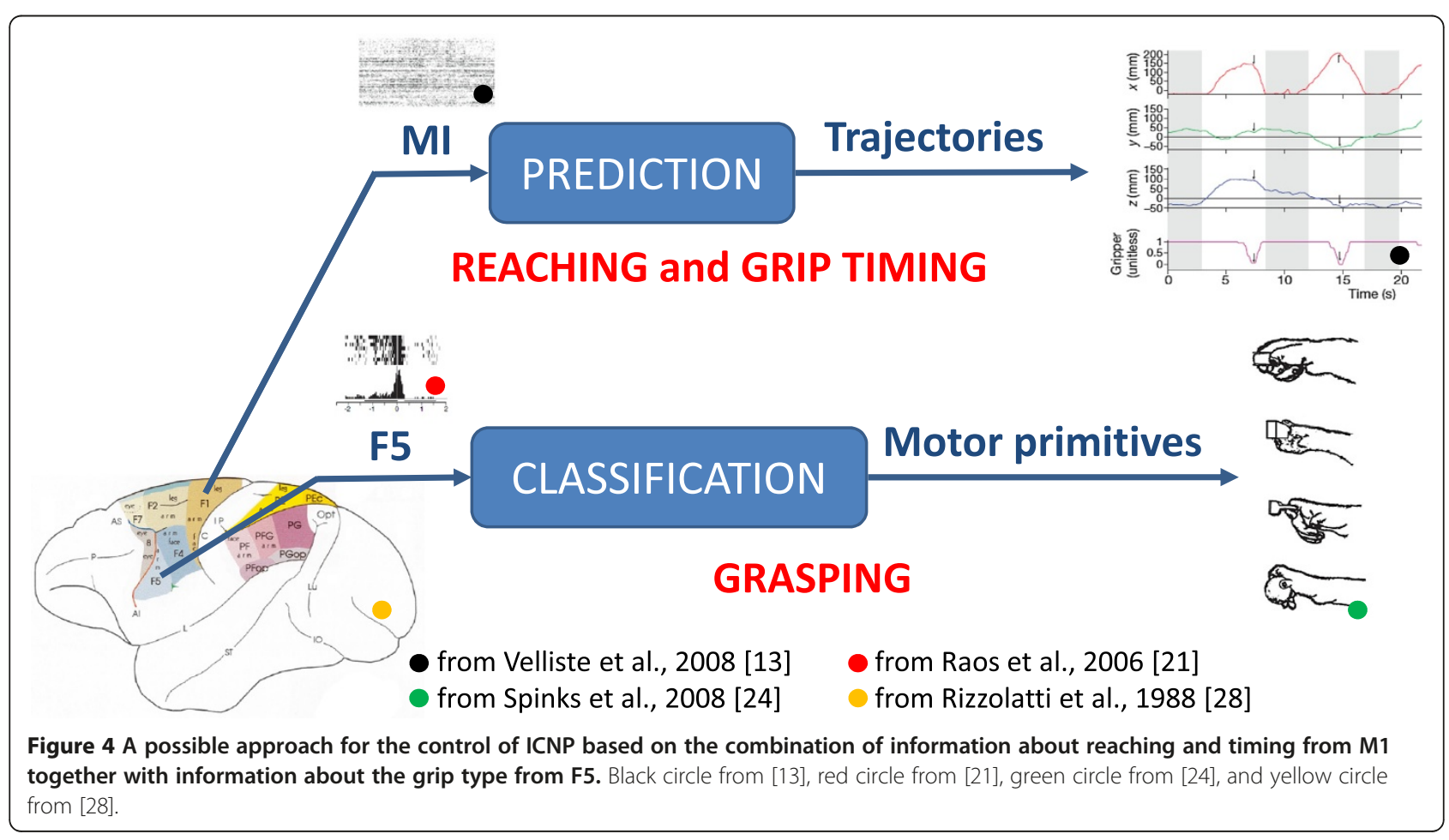


computer cursors and robotic devices but also to select actions or grips could represent a real improvement of functionality for neuroprosthetic devices.

\section{Abbreviations}

ICNPS: Invasive cortical neuroprostheses; M1: Primary motor cortex; nFR: Normalized firing rate; RR: Recognition ratio; SVMs: Support vector machines.

\section{Competing interests}

The authors declare that they have no competing interests.

\section{Authors' contributions}

JC and SM developed the decoding algorithm. VR, MAU, LF, AM, and VG designed the experimental protocol and performed the animal experiments. All authors wrote, read and approved the final manuscript.

\section{Acknowledgements}

This work was partially supported by the EU within the NEUROBOTICS Integrated Project (IST-FET Project 2003-001917: The fusion of NEUROscience and $\mathrm{rOBOTICS}$ )

\section{Author details}

${ }^{1}$ Neural Engineering Area, The BioRobotics Institute, Scuola Superiore Sant'Anna, Pisa, Italy. ${ }^{2}$ Department of Basic Sciences, Faculty of Medicine, School of Health Sciences, University of Crete, Iraklion, Greece. ${ }^{3}$ Foundation for Research and Technology-Hellas, Institute of Applied and Computational Mathematics, Iraklion, Greece. ${ }^{4}$ Department of Neuroscience, Section of Physiology, University of Parma, Parma, Italy. ${ }^{5}$ Italian Institute of Technology, RTM, Parma, Italy. ${ }^{6}$ Department of Psychology, University of Parma, Parma, Italy. ${ }^{7}$ Department of Physiology, Kinki University Faculty of Medicine, Osaka-Sayama, Japan. ${ }^{8}$ Translational Neural Engineering Lab, Center for Neuroprosthetics and Institute of Bioengineering, Ecole Polytechnique Federale de Lausanne, Lausanne, Switzerland.

Received: 24 February 2012 Accepted: 19 November 2012 Published: 26 November 2012

\section{References}

1. Micera S, Carpaneto J, Raspopovic S: Control of hand prostheses using peripheral information. Biomed Eng, IEEE Rev 2010, 3:48-68.

2. Hatsopoulos NG, Donoghue JP: The science of neural interface systems. Annu Rev Neurosci 2009, 32:249-266.

3. Ohnishi K, Weir RF, Kuiken TA: Neural machine interfaces for controlling multifunctional powered upper-limb prostheses. Expert Rev Med Dev 2007 4:43-53

4. Grill WM, Norman SE, Bellamkonda RV: Implanted neural interfaces: biochallenges and engineered solutions. Annu Rev Biomed Eng 2009, 11:1-24.

5. Navarro X, Krueger TB, Lago N, Micera S, Stieglitz T, Dario P: A critical review of interfaces with the peripheral nervous system for the control of neuroprostheses and hybrid bionic systems. J Peripher Nerv Syst 2005, 10:229-258

6. Dhillon GS, Horch KW: Direct neural sensory feedback and control of a prosthetic arm. IEEE Trans Neural Syst Rehabil Eng 2005, 13:468-472.

7. Micera S, Rossini PM, Rigosa J, Citi L, Carpaneto J, Raspopovic S, Tombini M, Cipriani C, Assenza G, Carrozza MC, et al: Decoding of grasping information from neural signals recorded using peripheral intrafascicular interfaces. J Neuroeng Rehabil 2011, 8:53.

8. Kim SP, Simeral JD, Hochberg LR, Donoghue JP, Friehs GM, Black MJ: Pointand-click cursor control with an intracortical neural interface system by humans with tetraplegia. IEEE Trans Neural Syst Rehabil Eng 2011, 19:193-203.

9. Hochberg LR, Serruya MD, Friehs GM, Mukand JA, Saleh M, Caplan AH, Branner A, Chen D, Penn RD, Donoghue JP: Neuronal ensemble control of prosthetic devices by a human with tetraplegia. Nature 2006, 442:164-171.

10. O'Doherty JE, Lebedev MA, lfft PJ, Zhuang KZ, Shokur S, Bleuler H, Nicolelis MA: Active tactile exploration using a brain-machine-brain interface. Nature 2011, 479:228-231.
11. Fetz EE: Volitional control of neural activity: implications for braincomputer interfaces. J Physiol 2007, 579:571-579.

12. Scherberger $\mathrm{H}$ : Neural control of motor prostheses. Curr Opin Neurobiol 2009, 19:629-633.

13. Velliste M, Perel S, Spalding MC, Whitford AS, Schwartz AB: Cortical control of a prosthetic arm for self-feeding. Nature 2008, 453:1098-1101.

14. Shenoy KV, Kaufman MT, Sahani M, Churchland MM: A dynamical systems view of motor preparation Implications for neural prosthetic system design. Prog Brain Res 2011, 192:33-58.

15. Hochberg LR, Bacher D, Jarosiewicz B, Masse NY, Simeral JD, Vogel J, Haddadin S, Liu J, Cash SS, van der Smagt P, Donoghue JP: Reach and grasp by people with tetraplegia using a neurally controlled robotic arm. Nature 2012, 485:372-375

16. Moritz CT, Perlmutter SI, Fetz EE: Direct control of paralysed muscles by cortical neurons. Nature 2008, 456:639-642.

17. Ethier C, Oby ER, Bauman MJ, Miller LE: Restoration of grasp following paralysis through brain-controlled stimulation of muscles. Nature 2012, 485:368-371.

18. Saleh M, Takahashi K, Amit Y, Hatsopoulos NG: Encoding of coordinated grasp trajectories in primary motor cortex. J Neurosci 2010, 30:17079-17090.

19. Vargas-Irwin CE, Shakhnarovich G, Yadollahpour P, Mislow JM, Black MJ, Donoghue JP: Decoding complete reach and grasp actions from local primary motor cortex populations. J Neurosci 2010, 30:9659-9669.

20. Rizzolatti G, Camarda R, Fogassi L, Gentilucci M, Luppino G, Matelli M: Functional organization of inferior area 6 in the macaque monkey. II. Area F5 and the control of distal movements. Exp Brain Res 1988, 71:491-507.

21. Raos V, Umiltà MA, Murata A, Fogassi L, Gallese V: Functional properties of grasping-related neurons in the ventral premotor area F5 of the macaque monkey. J Neurophysiol 2006, 95:709-729.

22. Townsend BR, Subasi E, Scherberger H: Grasp movement decoding from premotor and parietal cortex. J Neurosci 2011, 31:14386-14398.

23. Pistohl T, Schulze-Bonhage A, Aertsen A, Mehring C, Ball T: Decoding natural grasp types from human ECoG. Neurolmage 2011, 59:248-260.

24. Spinks RL, Kraskov A, Brochier T, Umilta MA, Lemon RN: Selectivity for grasp in local field potential and single neuron activity recorded simultaneously from M1 and F5 in the awake macaque monkey. J Neurosci 2008, 28:10961-10971.

25. Carpaneto J, Umilta MA, Fogassi L, Murata A, Gallese V, Micera S, Raos V: Decoding the activity of grasping neurons recorded from the ventral premotor area F5 of the macaque monkey. Neuroscience 2011, 188:80-94.

26. Hodges PW, Bui BH: A comparison of computer-based methods for the determination of onset of muscle contraction using electromyography. Electroencephalogr Clin Neurophysiol 1996, 101:511-519.

27. Chang C-C, Lin C-J: LIBSVM: A library for support vector machines. ACM Trans Int Syst Technol 2011, 2:1-27.

28. Rizzolatti G, Luppino G, Matelli M: The organization of the cortical motor system: new concepts. Electoencephalogr Clin Neurophysiol 1998, 106:283-296.

29. Farrell TR, Weir RF: The optimal controller delay for myoelectric prostheses. IEEE Trans Neural Syst Rehabil Eng 2007, 15:111-118.

30. Ganguly K, Dimitrov DF, Wallis JD, Carmena JM: Reversible large-scale modification of cortical networks during neuroprosthetic control. Nat Neurosci 2011, 14:662-667.

doi:10.1186/1743-0003-9-84

Cite this article as: Carpaneto et al:: Continuous decoding of grasping tasks for a prospective implantable cortical neuroprosthesis. Journal of NeuroEngineering and Rehabilitation 2012 9:84. 